\title{
Preparation and Enhancement of Thermal Conductivity of Heat Transfer Oil-Based $\mathrm{MoS}_{2}$ Nanofluids
}

\author{
Yuan-Xian Zeng, ${ }^{1}$ Xiu-Wen Zhong, ${ }^{1}$ Zhao-Qing Liu, ${ }^{1}$ Shuang Chen, ${ }^{2}$ and Nan Li ${ }^{1}$ \\ ${ }^{1}$ School of Chemistry and Chemical Engineering, Guangzhou Key Laboratory for Environmentally Functional Materials and \\ Technology, Guangzhou University, Guangzhou 510006, China \\ ${ }^{2}$ Guangzhou Institute of Railway Technology, Guangzhou 510430, China
}

Correspondence should be addressed to Nan Li; nanli518@126.com

Received 13 November 2013; Accepted 8 December 2013

Academic Editor: Wenjie Mai

Copyright (c) 2013 Yuan-Xian Zeng et al. This is an open access article distributed under the Creative Commons Attribution License, which permits unrestricted use, distribution, and reproduction in any medium, provided the original work is properly cited.

\begin{abstract}
The lipophilic $\mathrm{MoS}_{2}$ nanoparticles are synthesized by surface modification with stearic acid (SA). The heat transfer oil-based nanofluids, with the mass fraction of lipophilic nanoparticles varying from $0.25 \%$ up to $1.0 \%$, are prepared and their thermal conductivity is determined at temperatures ranging from 40 to $200^{\circ} \mathrm{C}$ using an apparatus based on the laser flash method. It has been found that the nanofluids have higher thermal conductivity and the thermal conductivity enhancement increased not only with increasing mass fraction of nanoparticles, but also with increasing temperature in the range $40-180^{\circ} \mathrm{C}$ The results show a $38.7 \%$ enhancement of the thermal conductivity of $\mathrm{MoS}_{2}$ nanofluid with only $1.0 \%$ mass fraction at $180^{\circ} \mathrm{C}$.
\end{abstract}

\section{Introduction}

Heat transfer fluids, such as water, ethylene glycol, and mineral oil, play important roles in many thermal transport applications. Their low thermal conductivity is one of the limits of the traditional heat transfer fluids. In order to improve the low thermal characteristics of the conventional heat transfer fluids, solid nanoparticles are added to form nanofluids, which were first introduced by Choi [1]. Enhanced thermophysical properties are the main favorable characteristics of nanofluids based on the fact that most solids have much higher thermal conductivities than common fluids. In the past decade, enhanced thermal conductivities of the water or ethylene glycol-based nanofluids containing a small amount of metals, metal oxides, nonmetallic materials, and polymers have been reported [2-8]. Currently many efforts have been focused on the oil-based nanofluids because of the needs in many industry fields to develop heat transfer fluids with preferred heat transfer properties in high temperature. Various oil-based nanofluids with silicon oil, diathermic oil, engine oil, and heat transfer oil used as the based fluid have been prepared [9-12].
However, the nanoparticles are easily sedimented after being added to the oil because of the poor compatibility between the nanoparticles and the base oil. The dispersion of nanoparticles in oils is still a principal problem for the application of nanofluids. In order to obtain better dispersion, an appropriate lipophilic modification process can be employed to improve the compatibility between the nanoparticles and the oil-based fluid. The organic ligands with long hydrocarbon chains were linked to the surface of the nanoparticles, and the surface-modified nanoparticles possess good dispersion behavior in oils. So far, most of these oilsoluble nanoparticles are investigated as lubricating oil additives [13-16], and only a few of them are used to prepare nanofluids for thermal conductivity enhancement $[17,18]$.

Molybdenum disulfide $\left(\mathrm{MoS}_{2}\right)$ has a layered structure and has been known and used as a lubricant and catalyst for several years because of its unique properties such as anisotropy, chemically inertness, and photocorrosion resistance [19]. The $\mathrm{MoS}_{2}$ nanoparticles, which are capped by dialkyldithiophosphate, have been synthesized and exhibit good lubrication properties [20, 21]. The sodium oleate and cetylamine also have been used together as the surface 


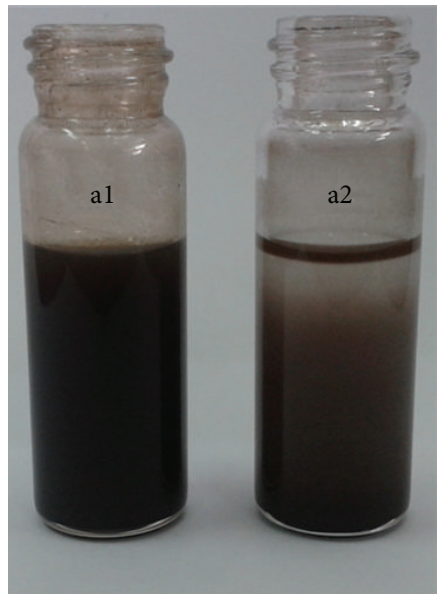

(a)

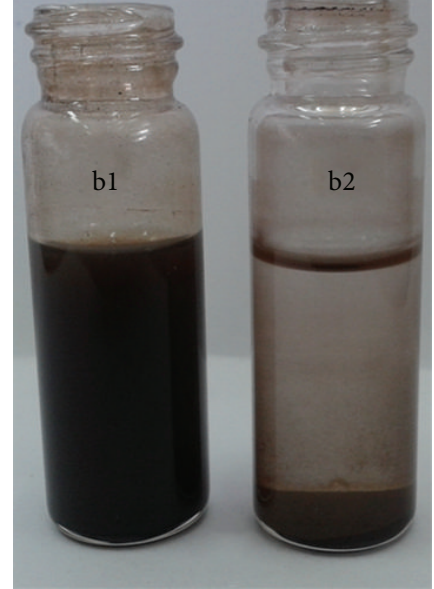

(b)

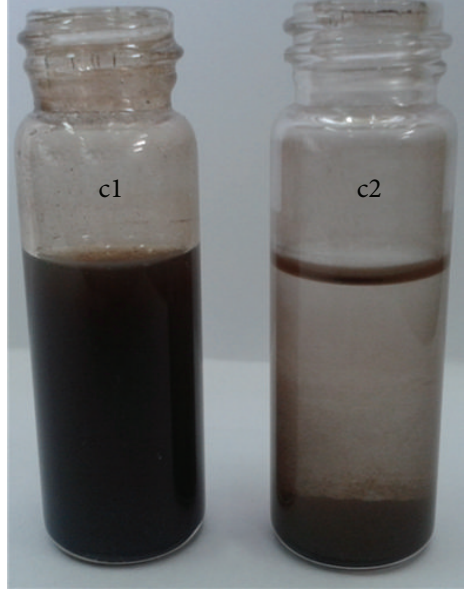

(c)

FIGURE 1: Sedimentation behavior of $0.5 \mathrm{wt} \% \mathrm{MoS}_{2}$ and SA-MoS ${ }_{2}$ nanoparticles in cyclohexane: (a) stay $10 \mathrm{~min}$, (b) stay $1 \mathrm{~h}$, and (c) stay $48 \mathrm{~h}$ (a1, b1, c1: SA-MoS 2 nanoparticles; a2, b2, c2: bare $\mathrm{MoS}_{2}$ nanoparticles).

modified reagents, and the oil-soluble $\mathrm{MoS}_{2}$ nanoparticles product can be directly used as hydrogenation nanocatalysts [22]. Nevertheless, to the best of our knowledge, no studies have been reported on the increased thermal conductivity of $\mathrm{MoS}_{2}$ nanofluids. Furthermore, the heat transfer oil is the preferred heat transfer medium in high temperature. So it is worthwhile to prepare and investigate heat transfer oil-based nanofluids containing $\mathrm{MoS}_{2}$ nanoparticles.

In the present work, $\mathrm{MoS}_{2}$ nanoparticles capped by stearic acid $\left(\mathrm{SA}-\mathrm{MoS}_{2}\right)$ are synthesized via a simple solution method. And the heat transfer oil-based nanofluids are prepared by dispersing these modified $\mathrm{MoS}_{2}$ nanoparticles with dibenzyl toluene as base fluid. The as-prepared nanofluids exhibit higher thermal conductivity compared to the base oil.

\section{Experimental}

2.1. Chemicals and Materials. Hydrazine hydrate (80\%) was purchased from Shanghai Aladdin Chemical Regent Co., Ltd (China). Heat transfer oil B350, which is one of the trade names of dibenzyl toluene, was obtained from Shanghai Ethylene Chemical Co. Ltd (China). All other reagents were of research grade or better and were obtained from commercial sources.

2.2. Synthesis of $\mathrm{MoS}_{2}$ Nanoparticles. The surface-modified $\mathrm{MoS}_{2}$ nanoparticles were prepared as follows: $\mathrm{Na}_{2} \mathrm{MoO}_{4}$. $2 \mathrm{H}_{2} \mathrm{O}(5.0 \mathrm{mmol})$ and $3.0 \mathrm{mmol}$ stearic acid were dissolved in $25.0 \mathrm{~mL}$ distilled water and $25.0 \mathrm{~mL}$ absolute alcohol at $80^{\circ} \mathrm{C}$ in a $250 \mathrm{~mL}$ three-necked flask. Then $0.6 \mathrm{~mL} \mathrm{~N} \mathrm{H}_{4} \cdot \mathrm{H}_{2} \mathrm{O}$ was added into the reaction flask and kept at that temperature for $1 \mathrm{~h}$. After that the hydrochloric acid $(1.0 \mathrm{~mol} / \mathrm{L})$ was added slowly into the reaction solution to adjust the $\mathrm{pH}$ value below 1.0 and stirred for $6 \mathrm{~h}$. Then the temperature fell to $60^{\circ} \mathrm{C}$, and $75.0 \mathrm{~mL}$ sodium sulfide solution $(0.20 \mathrm{~mol} / \mathrm{L})$ was dropped slowly into the reaction solution and reacted for $8 \mathrm{~h}$. The products were filtered, washed with hot ethanol, and then dried overnight in a vacuum at $60^{\circ} \mathrm{C}$.

The nonmodified $\mathrm{MoS}_{2}$ nanoparticles were prepared by the same method only without stearic acid added.

2.3. Preparation of Nanofluids. The $\mathrm{MoS}_{2}$ nanofluids were prepared by dispersing the surface-modified $\mathrm{MoS}_{2}$ nanoparticles in heat transfer oil B350 as base liquid. The samples were homogenized for about 5 min by ultrasonic vibration to ensure proper dispersion of the nanoparticles.

2.4. Characterization and Measurements. The phase analysis of surface-modified $\mathrm{MoS}_{2}$ was conducted by powder X-ray diffraction (XRD, Bruker, D8 ADVANCE) with $\mathrm{Cu}-\mathrm{K} \alpha$ radiation. The morphology of $\mathrm{MoS}_{2}$ was analyzed by using a JEOL JSM-7001F field emission scanning electron microscope (FESEM). A Nicolet 6700 ESP Fourier transform infrared spectrometer was used for FT-IR spectrum recording of the samples.

The long-term stability of $\mathrm{MoS}_{2}$ nanoparticles in organic solvents was characterized by the sediment test. The $\mathrm{MoS}_{2}$ nanoparticles which were introduced to cyclohexane and heat transfer oil B350, respectively, underwent ultrasonic vibration for about $10 \mathrm{~min}$ and were allowed to stand for more than 20 days. The thermal conductivity of $\mathrm{MoS}_{2}$ nanofluids was measured based on the laser flash method using the LFA447 Thermal Conductivity Properties Analyzer (NETZSCH, Germany).

\section{Results and Discussion}

3.1. Characterization of $\mathrm{MoS}_{2}$ Nanoparticles. The results of sedimentation test of two types of $\mathrm{MoS}_{2}$ nanoparticles suspended in cyclohexane are shown in Figure 1. Solution containing bare $\mathrm{MoS}_{2}$ nanoparticles has high settling rate and 


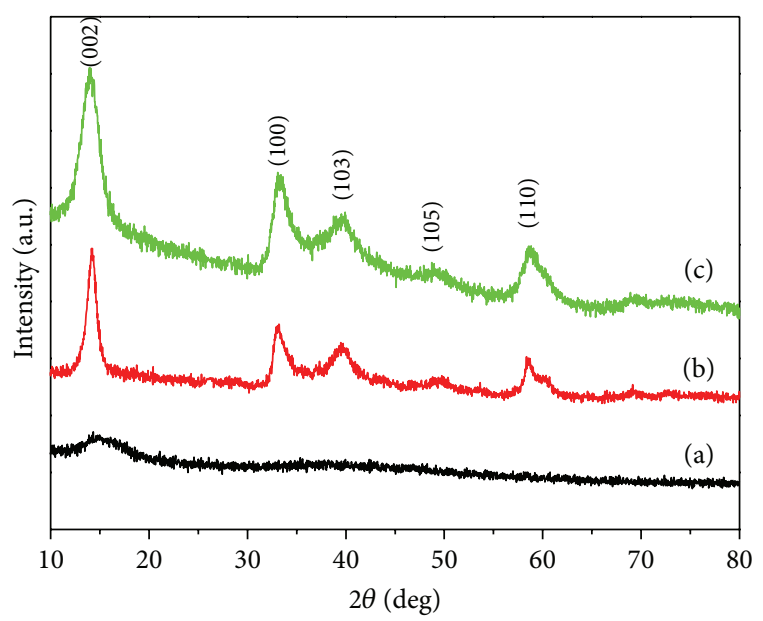

FIGURE 2: XRD patterns of (a) $\mathrm{MoS}_{2}$ nanoparticles without being annealed, (b) the samples annealed at $600^{\circ} \mathrm{C}$ for $1 \mathrm{~h}$, and (c) the samples annealed at $800^{\circ} \mathrm{C}$ for $1 \mathrm{~h}$.

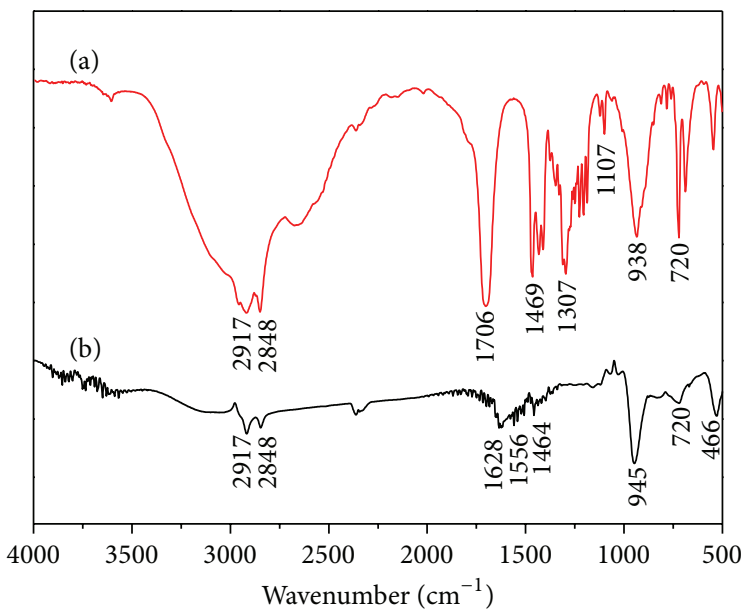

FIGURE 3: FT-IR spectrum of the stearic acid (a) and stearic acid modified $\mathrm{MoS}_{2}$ nanoparticles (b).

became transparent in less than $1 \mathrm{~h}$. Solution containing SA$\mathrm{MoS}_{2}$ nanoparticles has much lower settling rate and remains in the supernatant for a long time, which illuminates that grafting long carbon chain molecule on the $\mathrm{MoS}_{2}$ nanoparticles could significantly improve its stability in organic solvent. SA-MoS 2 nanoparticles exhibit better dispersion behavior in heat transfer oil because of the higher viscosity. The solution remains turbid even after 20 days.

Figure 2 shows the X-ray diffraction pattern of SA- $\mathrm{MoS}_{2}$ nanoparticles. Figure 2(a) gives only a broad weak peak (002) in its characteristic region $2 \theta=14.2^{\circ}$, which is the most important peak while other diffractions planes appeared in smaller intensities. Figure 2(a) indicates that the obtained $\mathrm{MoS}_{2}$ products are amorphous with poor crystals and highly disordered layered structure, which is consistent with the earlier reports $[23,24]$. This can be explained by the randomness and the tensile force of the crystals and only a few layers of $\mathrm{MoS}_{2}$ stack along this direction. It has been reported that the crystallinity of the $\mathrm{MoS}_{2}$ prepared by liquid precipitation method could be improved by annealing at high temperature [25]. Figures 2(b) and 2(c) give the XRD patterns of the prepared $\mathrm{MoS}_{2}$ samples which were annealed for $1 \mathrm{~h}$ under the flow of $\mathrm{N}_{2}$ at $600^{\circ} \mathrm{C}$ and $800^{\circ} \mathrm{C}$, respectively. In Figures 2(b) and 2(c), with the annealing temperature increasing from $600^{\circ} \mathrm{C}$ to $800^{\circ} \mathrm{C}$, the peaks of (002), (100), (103), (105), and (110) became high and sharp increasingly, which indicates that the crystals of $\mathrm{MoS}_{2}$ samples are much thicker and the well-stacked layered structure of $\mathrm{MoS}_{2}$ formed when the $\mathrm{MoS}_{2}$ samples were roasted. And the diffraction peaks can be readily indexed to the hexagonal phase of $\mathrm{MoS}_{2}$ (JCPDS no. $37-1492$ ) with lattice constants $a=0.3161 \mathrm{~nm}, b=0.3161 \mathrm{~nm}$, and $c=1.2299 \mathrm{~nm}$. The above analysis results also indicate that the crystallinity of the $\mathrm{MoS}_{2}$ samples could be usefully enhanced by the annealing.

Infrared spectra of stearic acid and surface-modified $\mathrm{MoS}_{2}$ nanoparticles are shown in Figure 3. As shown in Figure 3(a), the peaks at $2848 \mathrm{~cm}^{-1}$ and $2917 \mathrm{~cm}^{-1}$ are assigned to the stretching vibrations of $-\mathrm{CH}_{2}$ groups. The peak at around $1706 \mathrm{~cm}^{-1}$ belongs to carbonyl stretch of stearic acid, which disappeared in the spectrum of SA- $\mathrm{MoS}_{2}$ (Figure 3(b)), indicating that the chemical reaction took place between stearic acid and $\mathrm{MoS}_{2}$. As shown in Figure 3(b), the new appearance of two characteristic peaks at $1628 \mathrm{~cm}^{-1}$ and $1556 \mathrm{~cm}^{-1}$ is observed which belong to the symmetric and asymmetric carboxylate group stretching band, $v(\mathrm{COO}-)_{\text {asym }}$ and $v(\mathrm{COO}-)_{\text {sym }}$, respectively [26]. All above results show that the stearic acid has reacted with $\mathrm{MoS}_{2}$ nanoparticles and formed new chemical bonds, and the carboxylate radical of stearic acid did not dissociate after the reaction of stearic acid with $\mathrm{MoS}_{2}$ nanoparticles. So it is found that the disappearance of characteristic peaks of carboxyl $(-\mathrm{COOH})$ of stearic acid in Figure 3(b) confirms the formation of a monomolecular layer of stearic acid surrounding the $\mathrm{MoS}_{2}$ nanoparticle surface. Because of numerous stearic acid coated onto the surface of $\mathrm{MoS}_{2}$ nanoparticles, the long alkyl chain on the surface of $\mathrm{MoS}_{2}$ enhances lipophilicity of products greatly. So the SA- $\mathrm{MoS}_{2}$ nanoparticles are very soluble in heat transfer oils to easily form stable nanofluids. In addition, the coating layers should not easily separate from the surface of the $\mathrm{MoS}_{2}$ nanoparticles when the SA-MoS 2 nanoparticles are dispersed in the oil-based fluids.

Figure 4 shows SEM image of nonmodified $\mathrm{MoS}_{2}$ nanoparticles (Figure 4(a)) and SEM image of SA- $\mathrm{MoS}_{2}$ nanoparticles (Figure 4(b)). It was found in Figure 4(a) that nonmodified $\mathrm{MoS}_{2}$ nanoparticles tended to agglomerate and did not permit identification of a single particle, owing to the high surface energy. Figure 4(b) shows that the SA-MoS samples are not quite inerratic spherical shape nanoparticles with diameter in the range of 50-100 nm. Comparing Figures 4(a) and 4(b), it can be concluded that the existence of the surface modification layer effectively prevented agglomeration among $\mathrm{MoS}_{2}$ particles and led to the formation of fine nanoparticles.

3.2. Thermal Conductivities of Nanofluids. Figure 5 shows the effect of temperature on the enhancement of thermal 


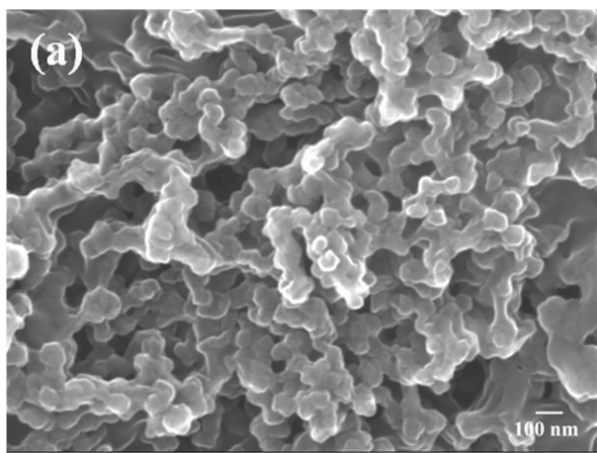

(a)

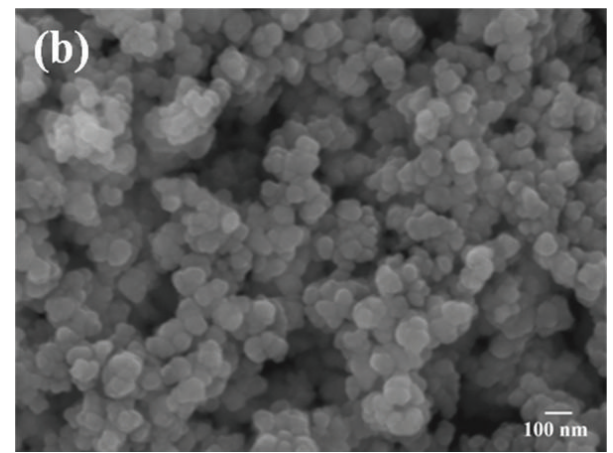

(b)

FIGURE 4: SEM images of nonmodified $\mathrm{MoS}_{2}$ nanoparticles (a) and $\mathrm{SA}-\mathrm{MoS}_{2}$ nanoparticles (b).

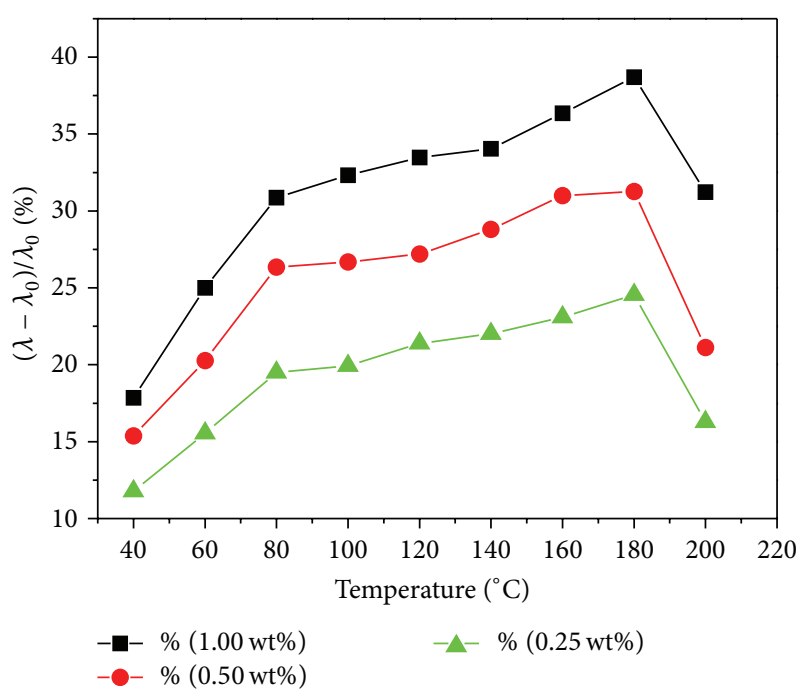

FIGURE 5: Thermal conductivity enhancements of nanofluids with different concentrations as a function of temperatures.

conductivity of $\mathrm{MoS}_{2}$ nanofluids, where $\lambda$ and $\lambda_{0}$ are the thermal conductivity of the nanofluids and base oil at various temperatures, respectively. Heat transfer oil B350 is used as base fluid. The thermal conductivity of nanofluids with 0.25 , 0.50 , and $1.0 \%$ mass fractions was measured. All the thermal conductivity of nanofluids was detected at different temperatures ranging from 40 to $200^{\circ} \mathrm{C}$, with steps of $20^{\circ} \mathrm{C}$.

The results show that both the heat transfer oil-based $\mathrm{MoS}_{2}$ nanofluids are found to have higher thermal conductivity enhancement at a much lower fraction of nanoparticles. A maximum $38.7 \%$ increase in thermal conductivity can be observed with respect to base oil at only $1.0 \%$ mass fraction with heat transfer oil B350 used as base oil. The effective thermal conductivity of heat transfer oil B350-based nanofluids increases as the mass fraction increases. These results show good agreement with the measured data of other oil-based nanofluids $[10,17,18]$. And this behavior can be explained with the increased particle-to-particle interactions at higher mass fraction because the distance between nanoparticles decreases [18].
The thermal conductivity enhancement of heat transfer oil B350-based nanofluids significantly increases with increasing temperature when the temperature is lower than $180^{\circ} \mathrm{C}$. This tendency is consistent with the previously reported results which are all measured at the temperature below $80^{\circ} \mathrm{C}[9,27,28]$. Actually, to the best of our knowledge, the thermal conductivity measurement of oil-based nanofluid at high temperature (more than $100^{\circ} \mathrm{C}$ ) has rarely been reported yet. The most probable explanation for this sensitivity to temperature is that the Brownian motion of nanoparticles is more intense at higher temperature [27]. However, the thermal conductivity enhancement decreases when the temperature is higher than $180^{\circ} \mathrm{C}$. This observation is probably attributed to vaporization of the organic oil because the flash point of dibenzyl toluene is $200^{\circ} \mathrm{C}$. So it can be suggested that the vaporization is aggravated when the temperature is close to the flash point. And the considerable vapor generated in the sample holder leads to the reducing of the measuring data, since the gas has lower thermal conductivity than that of liquid. This suggestion also is suitable for why the thermal conductivity enhancement increases sharply at low temperature (below $80^{\circ} \mathrm{C}$ ) but slowly at high temperature (above $80^{\circ} \mathrm{C}$ ). Anyway, the heat transfer oil-based $\mathrm{MoS}_{2}$ nanofluids exhibit fantastic enhancement of the thermal conductivity compared with base oil at high temperature.

\section{Conclusions}

The heat transfer oil-based $\mathrm{MoS}_{2}$ nanofluids have been prepared by dispersing stearic acid-modified $\mathrm{MoS}_{2}$ in heat transfer oil B350. The modified ligand is effective to improve the lipophilic property of $\mathrm{MoS}_{2}$ nanoparticles. Thermal conductivity measurements reveal that the thermal conductivity enhancement reaches up to $38.7 \%$ at a mass fraction of only $1.0 \%$ at $180^{\circ} \mathrm{C}$. And the thermal conductivities have been determined experimentally as a function of mass fraction and temperature. It has been found that the thermal conductivity increases with the mass fraction of nanoparticles. And the temperature variation has obvious effects on the thermal conductivity enhancement. Interestingly, the measured thermal conductivity enhancement decreases when the temperature 
is close to the flash point of the base oil. Anyway, the longterm stability and high thermal conductivity clearly identify the lipophilic $\mathrm{MoS}_{2}$ nanoparticles as a favorable additive in thermal energy engineering.

\section{Acknowledgments}

The authors acknowledge the financial support of this work by Natural Science Foundations of China (Grant no. 21306030), the Natural Science Foundations of Guangdong Province (Grant nos. S2012010009719 and S2013040015229), the Innovative Talents Cultivation Project of Guangdong Province (Grant no. LYM11096), the Science and Technology Project of Guangzhou (Grant no. 12C52011621), Scientific Research Project of Guangzhou Municipal Colleges and Universities (Grant no. 2012A064), and the National Undergraduate's Innovation and Entrepreneurship Training Program (Grant no. 201311078001).

\section{References}

[1] S. U. S. Choi, "Enhancing thermal conductivity of fluids with nanoparticles," in Developments and Applications of NonNewtonian Flows, D. A. Siginer and H. P. Wang, Eds., vol. 231, pp. 99-105, ASME FED, 1995.

[2] H. E. Patel, S. K. Das, T. Sundararajan, A. Sreekumaran Nair, B. George, and T. Pradeep, "Thermal conductivities of naked and monolayer protected metal nanoparticle based nanofluids: manifestation of anomalous enhancement and chemical effects," Applied Physics Letters, vol. 83, no. 14, pp. 2931-2933, 2003.

[3] X. F. Li, D. S. Zhu, X. J. Wang, N. Wang, J. W. Gao, and H. $\mathrm{Li}$, "Thermal conductivity enhancement dependent $\mathrm{pH}$ and chemical surfactant for $\mathrm{Cu}-\mathrm{H}_{2} \mathrm{O}$ nanofluids," Thermochimica Acta, vol. 469, no. 1-2, pp. 98-103, 2008.

[4] M. P. Beck, Y. Yuan, P. Warrier, and A. S. Teja, "The thermal conductivity of alumina nanofluids in water, ethylene glycol, and ethylene glycol + water mixtures," Journal of Nanoparticle Research, vol. 12, no. 4, pp. 1469-1477, 2010.

[5] W. Duangthongsuk and S. Wongwises, "Measurement of temperature-dependent thermal conductivity and viscosity of $\mathrm{TiO}_{2}$-water nanofluids," Experimental Thermal and Fluid Science, vol. 33, no. 4, pp. 706-714, 2009.

[6] L. Colla, L. Fedele, M. Scattolini, and S. Bobbo, "Water-based $\mathrm{Fe}_{2} \mathrm{O}_{3}$ nanofluid characterization: thermal conductivity and viscosity measurements and correlation," Advances in Mechanical Engineering, vol. 2012, Article ID 674947, 8 pages, 2012.

[7] Y. Ding, H. Alias, D. Wen, and R. A. Williams, "Heat transfer of aqueous suspensions of carbon nanotubes (CNT nanofluids)," International Journal of Heat and Mass Transfer, vol. 49, no. 1-2, pp. 240-250, 2006.

[8] T. B. Blake, S. B. Paul, C. B. Jeremiah, M. L. Charles, and L. D. Jim, "Nanodiamond nanofluids for enhanced thermal conductivity," Acs Nano, vol. 7, no. 4, pp. 3183-3189, 2013.

[9] L. Chen and H. Xie, "Silicon oil based multiwalled carbon nanotubes nanofluid with optimized thermal conductivity enhancement," Colloids and Surfaces A, vol. 352, no. 1-3, pp. 136140, 2009.
[10] G. Colangelo, E. Favale, A. de Risi, and D. Laforgia, "Results of experimental investigations on the heat conductivity of nanofluids based on diathermic oil for high temperature applications," Applied Energy, vol. 97, pp. 828-833, 2012.

[11] S. M. S. Murshed, "Simultaneous measurement of thermal conductivity, thermal diffusivity, and specific heat of nanofluids," Heat Transfer Engineering, vol. 33, no. 8, pp. 722-731, 2012.

[12] D. Ashtiani, M. A. Akhavan-Behabadi, and M. Fakoor Pakdaman, "An experimental investigation on heat transfer characteristics of multi-walled CNT-heat transfer oil nanofluid flow inside flattened tubes under uniform wall temperature condition," International Communications in Heat and Mass Transfer, vol. 39, pp. 1404-1409, 2012.

[13] S. Chen and N. Li, "Synthesis and characterization of DDPcoated PbO nanoparticles," Journal of Materials Chemistry, vol. 12, no. 4, pp. 1124-1127, 2002.

[14] S. Zheng, R. Guan, Q. Guo, and D. Su, “The design of surface modification of amorphous $\mathrm{ZrO}_{2}$ nanoparticles as a lubricant additive," Surface Review and Letters, vol. 14, no. 6, pp. 10471052, 2007.

[15] L. Zhang, H. Wan, L. Chen, H. Zhou, and J. Chen, "Surfacemodified $\mathrm{Sb}_{2} \mathrm{~S}_{3}$ nanoparticles and their tribological behaviors in liquid paraffin," Journal of Dispersion Science and Technology, vol. 32, no. 6, pp. 846-850, 2011.

[16] X. Xiong, Y. Kang, G. Yang, S. Zhang, L. Yu, and P. Zhang, "Preparation and evaluation of tribological properties of $\mathrm{cu}$ nanoparticles surface modified by tetradecyl hydroxamic acid," Tribology Letters, vol. 46, no. 3, pp. 211-220, 2012.

[17] D. Li, W. Xie, and W. Fang, "Preparation and properties of copper-oil-based nanofluids," Nanoscale Research Letters, vol. 6, article 373, 2011.

[18] B. Wang, B. Wang, P. Wei, X. Wang, and W. Lou, "Controlled synthesis and size-dependent thermal conductivity of $\mathrm{Fe}_{3} \mathrm{O}_{4}$ magnetic nanofluids," Dalton Transactions, vol. 41, no. 3, pp. 896-899, 2012.

[19] B. Pourabbas and B. Jamshidi, "Preparation of $\mathrm{MoS}_{2}$ nanoparticles by a modified hydrothermal method and the photocatalytic activity of $\mathrm{MoS}_{2} / \mathrm{TiO}_{2}$ hybrids in photo-oxidation of phenol," Chemical Engineering Journal, vol. 138, no. 1-3, pp. 5562,2008 .

[20] Z. J. Zhang, J. Zhang, and Q. J. Xue, "Synthesis and characterization of a molybdenum disulfide nanocluster," The Journal of Physical Chemistry, vol. 98, no. 49, pp. 12973-12977, 1994.

[21] L. Yu, P. Zhang, and Z. Du, "Tribological behavior and structural change of the LB film of $\mathrm{MoS}_{2}$ nanoparticles coated with dialkyldithiophosphate," Surface and Coatings Technology, vol. 130, no. 1, pp. 110-115, 2000.

[22] S. Wang, C. An, J. He, and Z. Wang, "Facile preparation of efficient oil-soluble $\mathrm{MoS}_{2}$ hydrogenation nanocatalysts," Journal of Natural Gas Chemistry, vol. 20, no. 4, pp. 408-412, 2011.

[23] Y. Tian, Y. He, and Y. Zhu, "Low temperature synthesis and characterization of molybdenum disulfide nanotubes and nanorods," Materials Chemistry and Physics, vol. 87, no. 1, pp. 87-90, 2004.

[24] G. Li, C. Li, H. Tang et al., "Synthesis and characterization of hollow $\mathrm{MoS}_{2}$ microspheres grown from $\mathrm{MoO}_{3}$ precursors," Journal of Alloys and Compounds, vol. 501, no. 2, pp. 275-281, 2010.

[25] X.-L. Li and Y.-D. Li, "MoS ${ }_{2}$ nanostructures: synthesis and electrochemical $\mathrm{Mg}^{2+}$ intercalation," The Journal of Physical Chemistry B, vol. 108, no. 37, pp. 13893-13900, 2004. 
[26] L. Zhang, L. Chen, H. Wan, J. Chen, and H. Zhou, "Synthesis and tribological properties of stearic acid-modified anatase $\left(\mathrm{TiO}_{2}\right)$ nanoparticles," Tribology Letters, vol. 41, no. 2, pp. 409416, 2011.

[27] B. Wang, X. Wang, W. Lou, and J. Hao, "Gold-ionic liquid nanofluids with preferably tribological properties and thermal conductivity," Nanoscale Research Letters, vol. 6, no. 1, pp. 259269, 2011.

[28] M. Saeedinia, M. A. Akhavan-Behabadi, and P. Razi, “Thermal and rheological characteristics of $\mathrm{CuO}$-Base oil nanofluid flow inside a circular tube," International Communications in Heat and Mass Transfer, vol. 39, no. 1, pp. 152-159, 2012. 

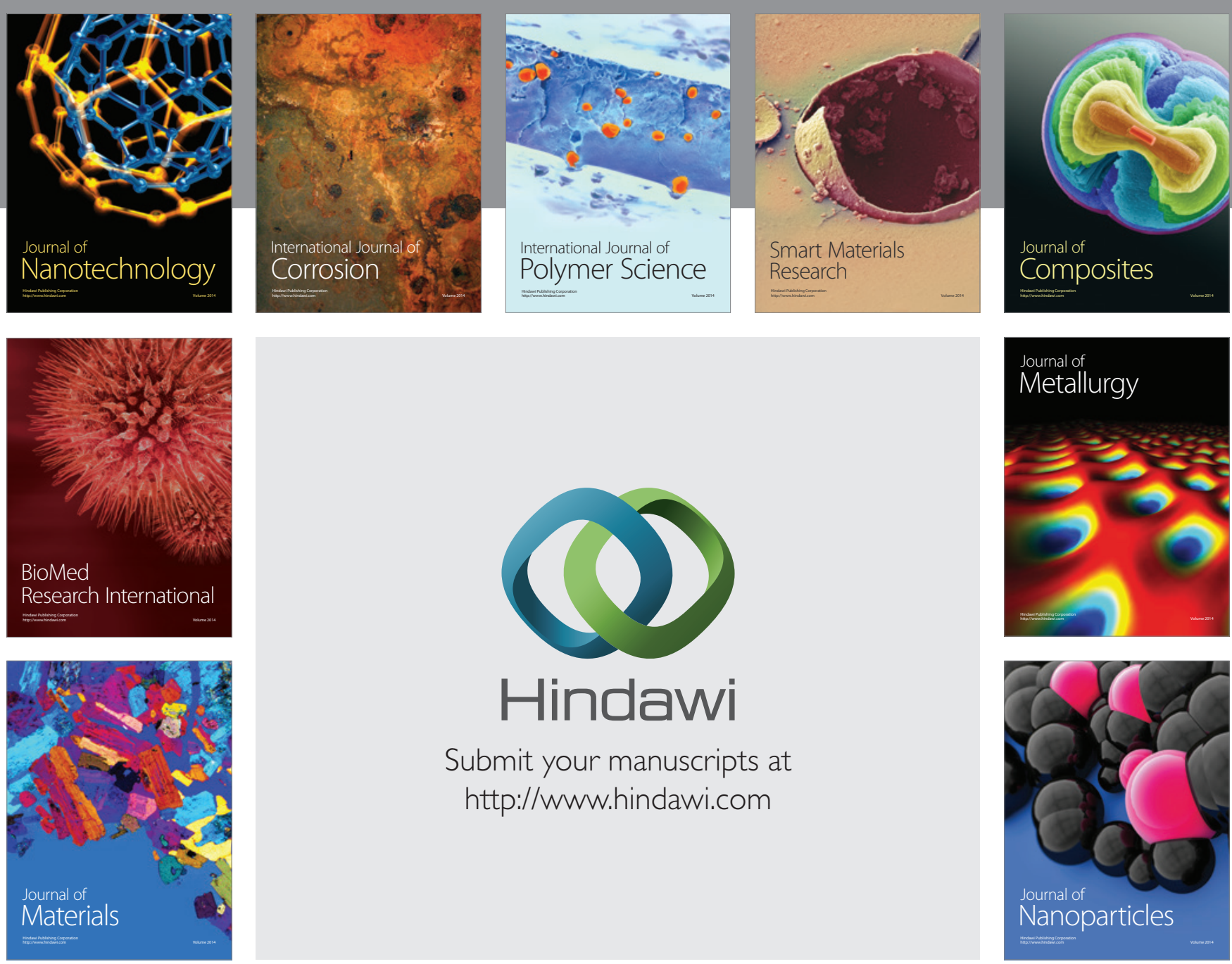

Submit your manuscripts at http://www.hindawi.com
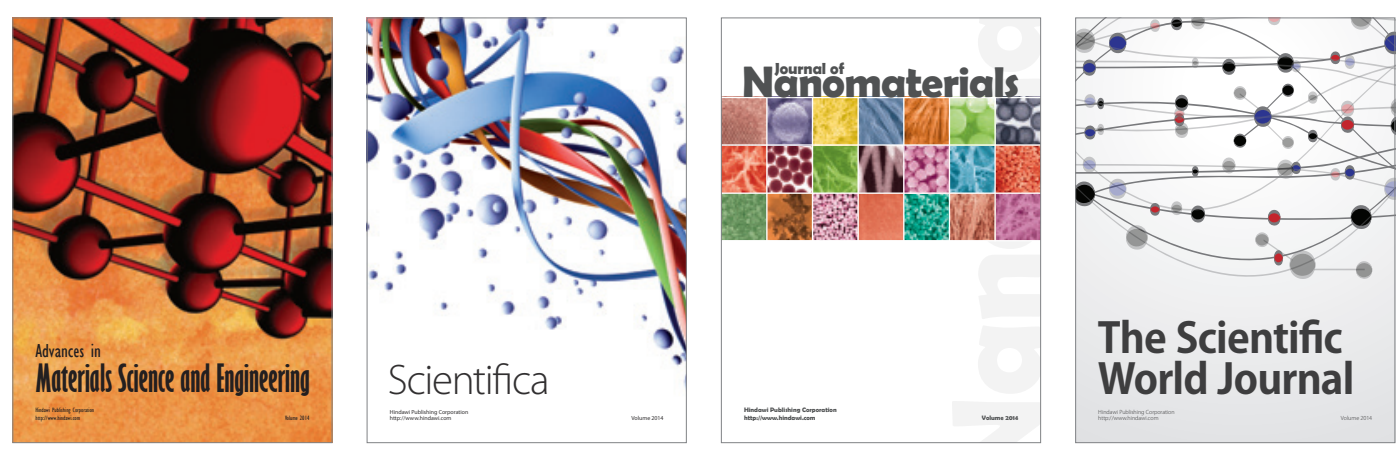

\section{The Scientific World Journal}
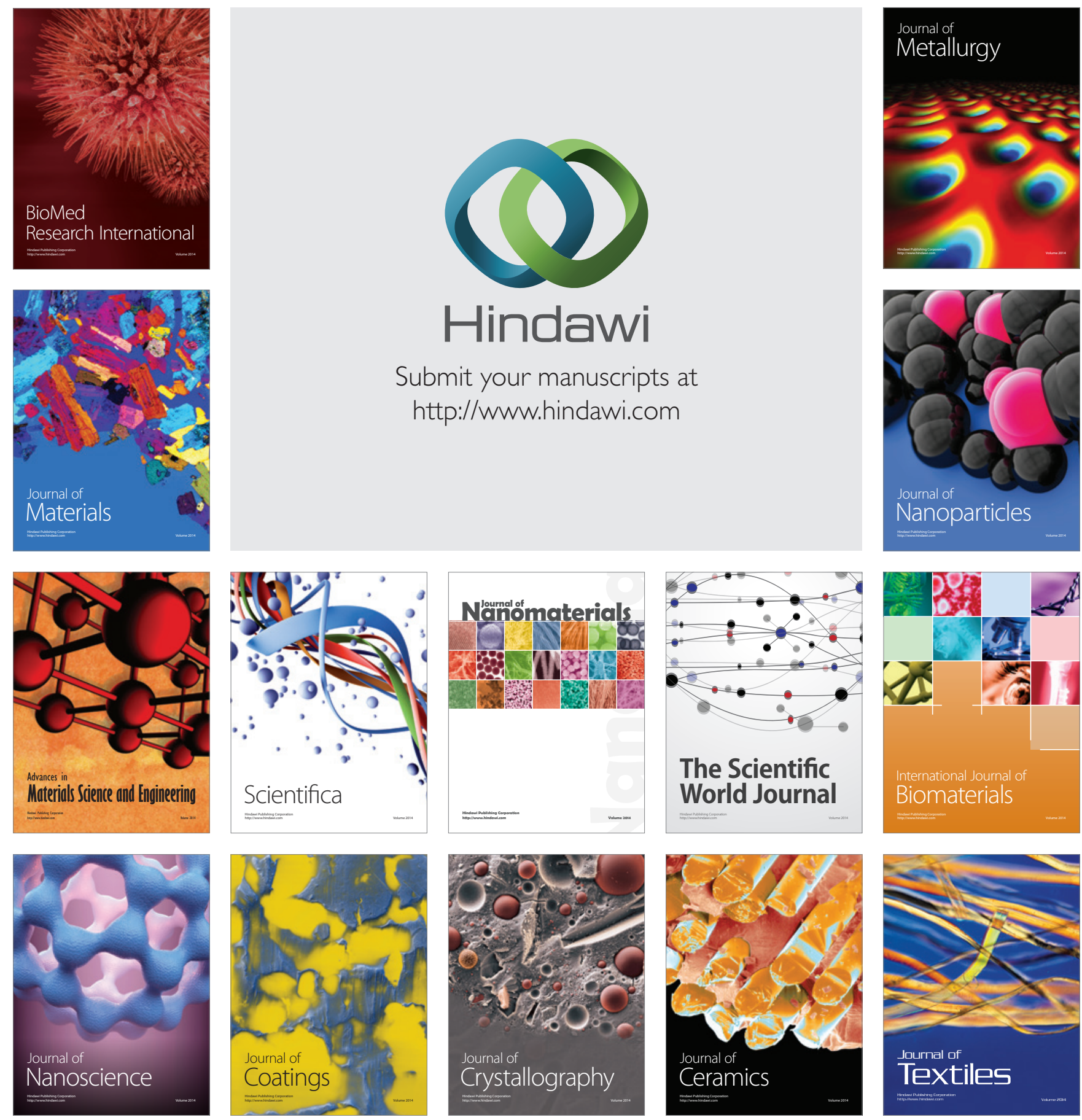